\title{
On forced oscillations in groups of interacting nonlinear systems
}

\author{
Ivan Polekhin
}

\begin{abstract}
Consider a periodically forced nonlinear system which can be presented as a collection of smaller subsystems with pairwise interactions between them. Each subsystem is assumed to be a massive point moving with friction on a compact surface, possibly with a boundary, in an external periodic field. We present sufficient conditions for the existence of a periodic solution for the whole system. The result is illustrated by a series of examples including a chain of strongly coupled pendulums in a periodic field.
\end{abstract}

Keywords: periodic solution, Euler-Poincaré characteristic, nonlinear system, coupled pendulums, nonlinear lattice

\section{Brief introduction}

The phenomenon of forced oscillations has been studied since at least 1922, when G. Hamel proved [1] that the equation describing the motion of a periodically forced pendulum have at least one periodic solution. Various results, which generalize and develop [1], have appeared in the literature since this paper; see, for example, [2, 3, 4, 5, 6]. Forced oscillations in a system of coupled planar pendulums and its generalizations has been studied in [7, 8] under the assumption of certain symmetry properties for the forcing terms. The existence and multiplicity of periodic solutions for coupled systems also discussed in [9].

At the same time, a nonlinear lattice is a cornerstone model in nonlinear physics and it is widely used for analytical and computational purposes. See, for example, [10, 11, 12, 13]. In our work we present a result which lies in the intersection of the both mentioned research areas and can be useful for studying forced oscillations in a nonlinear lattice and its generalizations. The result given in the paper continues a previously reported result [14].

In the paper the following systems are considered. Let us have several compact smooth manifolds, possibly with boundaries, with non-zero Euler-Poincaré characteristics. Suppose that for each manifold there is a massive point moving on it with viscous friction. All points are in an external periodic field and may interact with each other. It is allowed that the interactions may be of different types and also may be arbitrarily strong. We present sufficient conditions for the existence of a periodic solution for such a system.

Email address: ivanpolekhin@gmail.com (Ivan Polekhin) 
We would like to note that when the considered manifolds are closed, it isn't hard to prove that there exists a periodic solution in the system: it directly follows from the Lefschetz-Hopf theorem. Yet in applications it appears to be useful to consider manifolds with boundaries to prove the existence of a periodic solution and estimate it. One of the examples of such kind, which we consider further below, is a widely used in nonlinear studies model of coupled oscillators on a line. Therefore, our result generalizes the approach based on an application of the Lefschetz-Hopf theorem to the important case of compact manifolds with boundaries.

Our result is based on a theorem by R. Srzednicki, K. Wójcik, and P. Zgliczyński [15] and provides an illustrative geometrical approach to study periodical oscillations. Since the result is presented in a coordinate-free form, it also avoids possible shortcomings of purely analytical approaches and can be applied to the systems with complex topology of the phase space.

The main theorem is illustrated by a series of examples from mechanics including a system of an arbitrary number of strongly coupled pendulums in a periodic external field.

\section{Main result}

\subsection{Governing equations}

The equations introduced in this subsection — which we are going to use further below in the paper - generalize the governing equations for a mechanical system consisting of massive points moving with friction-like interaction on compact surfaces. For the sake of simplicity, we assume that all manifolds and considered functions are smooth (i.e. $C^{\infty}$ ).

Let $M_{i}$ be a compact connected one- or two-dimensional manifold (possibly with a boundary), where $i=1, \ldots, n$. For all $i=1, \ldots, n$, there is a point moving in an external force field on the manifold $M_{i}$. We also assume that there is a friction-like force acting on the point.

In our further consideration, we will study the behavior of the system in vicinities of $\partial M_{i}$ and it will be convenient to consider enlarged manifolds $M_{i}^{+}$. Let $M_{i}^{+}$be a boundaryless connected manifold such that $M_{i} \subset M_{i}^{+}, \operatorname{dim} M_{i}=\operatorname{dim} M_{i}^{+}$. Also suppose that every manifold $M_{i}^{+}$is equipped with a Riemannian metric $\langle\cdot, \cdot\rangle_{i}$.

If the points do not interact, one can consider the following independent equations of motion

$$
\nabla_{\dot{q}_{i}}^{i} \dot{q}_{i}=f_{i}\left(t, q_{i}, \dot{q}_{i}\right)+f_{i}^{\text {friction }}\left(t, q_{i}, \dot{q}_{i}\right), \quad i=1, \ldots, n,
$$

where $\nabla^{i}$ means the covariant differentiation with respect to the corresponding metric $\langle\cdot, \cdot\rangle_{i}$ for the $i$-th point moving on $M_{i}^{+} ; f_{i}: \mathbb{R} / T \mathbb{Z} \times T M_{i}^{+} \rightarrow T M_{i}^{+}$and $f_{i}\left(t, q_{i}, \dot{q}_{i}\right) \in T_{q_{i}} M_{i}^{+}$for any $t, q_{i}, \dot{q}_{i} ; f_{i}^{\text {friction }}: \mathbb{R} / T \mathbb{Z} \times T M_{i}^{+} \rightarrow T M_{i}^{+}$corresponds to a friction-like force.

As we said before, (1) includes the case of a mechanical system of massive points in an external field. Indeed, in this case $\langle\cdot, \cdot\rangle_{i}$ is the corresponding kinetic metric and, for given $t, q_{i}$ and $\dot{q}_{i}, f_{i}\left(t, q_{i}, \dot{q}_{i}\right)$ and $f_{i}^{\text {friction }}\left(t, q_{i}, \dot{q}_{i}\right)$ are the dual vectors to the corresponding generalized forces. Note that from (1) it follows that if there is no forces acting on the $i$-th point then (1) becomes $\nabla_{\dot{q}_{i}}^{i} \dot{q}_{i}=0$, which is the equation of the geodesic motion. 
In general form, when the interactions are took into account, we consider the following equations of motion

$$
\nabla_{\dot{q}_{i}}^{i} \dot{q}_{i}=f_{i}\left(t, q_{i}, \dot{q}_{i}\right)+f_{i}^{\text {friction }}\left(t, q_{i}, \dot{q}_{i}\right)+f_{i}^{\text {interaction }}\left(t, q_{1}, \dot{q}_{1}, \ldots, q_{n}, \dot{q}_{n}\right), \quad i=1, \ldots, n,
$$

where $f_{i}^{\text {interaction }}: \mathbb{R} / T \mathbb{Z} \times T M_{1}^{+} \times \ldots \times T M_{n}^{+} \rightarrow T M_{i}^{+}$and $f_{i}^{\text {interaction }}\left(t, q_{1}, \dot{q}_{1}, \ldots, q_{n}, \dot{q}_{n}\right) \in$ $T_{q_{i}} M_{i}^{+}$for any $t, q_{j}, \dot{q}_{j}, j=1, \ldots, n$. Here the functions $f_{i}^{\text {interaction }}$, being defined on $\mathbb{R} / T \mathbb{Z} \times$ $T M_{1}^{+} \times \cdots \times T M_{n}^{+}$, may describe more general forces than the pairwise interactions usually considered in applications.

As usual, we say that the system has a $T$-periodic solution if there are functions $q_{i}: \mathbb{R} \rightarrow M_{i}^{+}$ which satisfy (2) and $q_{i}(t+T)=q_{i}(t)$, for all $t \in[0, T), i=1, \ldots, n$.

Finally, rewrite (2) as follows

$$
\begin{aligned}
& \dot{q}_{i}=p_{i}, \\
& \nabla_{p_{i}}^{i} p_{i}=f_{i}\left(t, q_{i}, p_{i}\right)+f_{i}^{\text {friction }}\left(t, q_{i}, p_{i}\right)+f_{i}^{\text {interaction }}\left(t, q_{1}, p_{1}, \ldots, q_{n}, p_{n}\right), \quad i=1, \ldots, n .
\end{aligned}
$$

In the rest of the paper, we will assume that $f_{i}^{\text {friction }}, f_{i}$ and $f_{i}^{\text {interaction }}$ satisfy the following conditions:

(H1) There exist constants $d_{i}>0$ such that

$$
\sup _{\substack{t \in[0, T], q_{i} \in M_{i} \\\left\langle p_{i}, p_{i}\right\rangle_{i}>d_{i}}} \frac{\left\langle f_{i}^{\text {friction }}\left(t, q_{i}, p_{i}\right), p_{i}\right\rangle_{i}}{\left\langle p_{i}, p_{i}\right\rangle_{i}}<0, \quad i=1, \ldots, n .
$$

(H2) The functions $f_{i}$ and $f_{i}^{\text {interaction }}$ are bounded for all $i=1, \ldots, n$.

Remark 2.1. If we consider a mechanical system of massive points moving on surfaces in $\mathbb{R}^{3}$ with viscous friction with arbitrarily small friction coefficients then (4) is satisfied.

Lemma 2.2. Suppose that conditions (H1) and (H2) are satisfied, then for some $c_{i}>0$, $i=1, \ldots, n$ along the solutions of (3)

$$
\left.\frac{d}{d t} T_{i}\right|_{T_{i}=c_{i}}<0, \quad T_{i}=\left\langle p_{i}, p_{i}\right\rangle_{i}=\left\|p_{i}\right\|_{i}^{2}
$$

Proof. By direct calculation from (3), we have

$$
\begin{aligned}
\frac{d}{d t} T_{i} & =2\left\langle f_{i}\left(t, q_{i}, p_{i}\right)+f_{i}^{\text {friction }}\left(t, q_{i}, p_{i}\right)+f_{i}^{\text {interaction }}\left(t, q_{1}, p_{1}, \ldots, q_{n}, p_{n}\right), p_{i}\right\rangle_{i} \leqslant \\
& \leqslant 2\left\langle p_{i}, p_{i}\right\rangle_{i}\left(\frac{\left\|f_{i}\left(t, q_{i}, p_{i}\right)\right\|_{i}}{\left\|p_{i}\right\|_{i}}+\frac{\left\|f_{i}^{\text {interaction }}\left(t, q_{1}, p_{1}, \ldots, q_{n}, p_{n}\right)\right\|_{i}}{\left\|p_{i}\right\|_{i}}+\frac{\left\langle f_{i}^{\text {friction }}\left(t, q_{i}, p_{i}\right), p_{i}\right\rangle_{i}}{\left\langle p_{i}, p_{i}\right\rangle_{i}}\right) .
\end{aligned}
$$

Since $f_{i}$ and $f_{i}^{\text {interaction }}$ are bounded, then from (4) we obtain $d T_{i} / d t<0$ provided $c_{i}$ is large enough. 


\subsection{Auxiliary constructions and results}

The approach developed in [15] is based on the ideas of the Ważewski method [16] and the Lefschetz-Hopf theorem. In this subsection we introduce some definitions and a result from [15] which we slightly modify for our use.

Let $v: \mathbb{R} \times M \rightarrow T M$ be a time-dependent vector field on a manifold $M$

$$
\dot{x}=v(t, x)
$$

For $t_{0} \in \mathbb{R}$ and $x_{0} \in M$, the map $t \mapsto x\left(t, t_{0}, x_{0}\right)$ is the solution for the initial value problem for the system (5), such that $x\left(0, t_{0}, x_{0}\right)=x_{0}$. If $W \subset \mathbb{R} \times M, t \in \mathbb{R}$, then we denote

$$
W_{t}=\{x \in M:(t, x) \in W\} .
$$

Definition 2.3. Let $W \subset \mathbb{R} \times M$. Define the exit set $W^{-}$as follows. A point $\left(t_{0}, x_{0}\right)$ is in $W^{-}$if there exists $\delta>0$ such that $\left(t+t_{0}, x\left(t, t_{0}, x_{0}\right)\right) \notin W$ for all $t \in(0, \delta)$.

Definition 2.4. We call $W \subset \mathbb{R} \times M$ a Ważewski block for the system (5) if $W$ and $W^{-}$ are compact.

Definition 2.5. A set $W \subset[a, b] \times M$ is called a simple periodic segment over $[a, b]$ if it is a Ważewski block with respect to the system (5), $W=[a, b] \times Z$, where $Z \subset M$, and $W_{t_{1}}^{-}=W_{t_{2}}^{-}$for any $t_{1}, t_{2} \in[a, b)$.

Definition 2.6. Let $W$ be a simple periodic segment over $[a, b]$. The set $W^{--}=[a, b] \times W_{a}^{-}$ is called the essential exit set for $W$.

In our case, the result from [15] can be presented as follows.

Theorem 2.7. [15] Let $W$ be a simple periodic segment over $[a, b]$. Then the set

$$
U=\left\{x_{0} \in W_{a}: x\left(t-a, a, x_{0}\right) \in W_{t} \backslash W_{t}^{--} \text {for all } t \in[a, b]\right\}
$$

is open in $W_{a}$ and the set of fixed points of the restriction $\left.x(b-a, a, \cdot)\right|_{U}: U \rightarrow W_{a}$ is compact. Moreover, if $W_{a}$ and $W_{a}^{-}$are ANRs then the fixed point index of $\left.x(b-a, a, \cdot)\right|_{U}$ can be calculated by means of the Euler-Poincaré characteristic of $W$ and $W_{a}^{-}$as follows

$$
\operatorname{ind}\left(\left.x(b-a, a, \cdot)\right|_{U}\right)=\chi\left(W_{a}\right)-\chi\left(W_{a}^{-}\right) .
$$

In particular, if $\chi\left(W_{a}\right)-\chi\left(W_{a}^{-}\right) \neq 0$ then $\left.x(b-a, a, \cdot)\right|_{U}$ has a fixed point in $W_{a}$. 


\subsection{Main theorem}

In this subsection, we prove our main result and illustrate it with a series of examples. Below we use the following notations: $M=M_{1} \times \ldots \times M_{n}$ and $M^{+}=M_{1}^{+} \times \ldots \times M_{n}^{+}$.

Theorem 2.8. Suppose that for (3) the following conditions are satisfied

1. The Euler-Poincaré characteristic of $M_{i}$ is non-zero for all $i=1, \ldots, n$.

2. (H1) and (H2) are satisfied.

3. For any $t_{0} \in \mathbb{R},\left(q_{1}^{0}, p_{1}^{0}, \ldots, q_{n}^{0}, p_{n}^{0}\right) \in T(\partial M)$ there is an $\varepsilon>0$ such that

$$
q\left(t, t_{0}, q_{1}^{0}, p_{1}^{0}, \ldots, q_{n}^{0}, p_{n}^{0}\right) \notin M, \quad \text { for all } \quad t \in(0, \varepsilon) .
$$

Then there exists a solution $\left(q_{1}, p_{1}, \ldots, q_{n}, p_{n}\right): \mathbb{R} \rightarrow T M^{+}$of (3) such that for all $i=1, \ldots, n$

$$
q_{i}(t)=q_{i}(t+T), \quad p_{i}(t)=p_{i}(t+T), \quad q_{i}(t) \in M_{i} \backslash \partial M_{i}, \quad \text { for all } t \in \mathbb{R} .
$$

Proof. Consider the following compact subset $W$ of $[0, T] \times T M^{+}$

$W=\left\{0 \leqslant t \leqslant T,\left(q_{1}, p_{1}, \ldots, q_{n}, p_{n}\right) \in T M^{+}:\left(q_{1}, \ldots, q_{n}\right) \in M_{1} \times \ldots \times M_{n},\left\langle p_{i}, p_{i}\right\rangle_{i} \leqslant c_{i}, i=1, \ldots, n\right\}$

where $c_{i}>0$ are the constants obtained from lemma 2.2 .

From lemma 2.2 we also have that if $\left(t, q_{1}, p_{1}, \ldots, q_{n}, p_{n}\right) \in W^{--}$then $\left(q_{1}, \ldots, q_{n}\right) \in \partial M$. Since

$$
\partial M=\partial M_{1} \times M_{2} \times \ldots \times M_{n} \cup \ldots \cup M_{1} \times \ldots M_{n-1} \times \partial M_{n},
$$

then for $\left(t, q_{1}, p_{1}, \ldots, q_{n}, p_{n}\right) \in W^{--}$there exists $i$ such that $q_{i} \in \partial M_{i}$. Let $\nu_{q_{i}} \in T_{q_{i}} M_{i}^{+}$be a normal vector to $\partial M_{i}$ at point $q_{i} \in \partial M_{i}$ such that, for all $p_{i} \in T_{q_{i}} M_{i}^{+},\left\langle\nu_{q_{i}}, p_{i}\right\rangle_{i}>0$, the solution starting from $\left(t, q_{1}, p_{1}, \ldots, q_{i}, p_{i}, \ldots, q_{n}, p_{n}\right)$ at least locally leaves $M$. From the above definition of $\nu_{q_{i}}$, we obtain for any $j$

$$
\begin{aligned}
W^{--} \supset\left\{0 \leqslant t \leqslant T,\left(q_{1}, p_{1}, \ldots, q_{n}, p_{n}\right) \in T M^{+}:\right. \\
\\
\left.\quad\left(q_{1}, \ldots, q_{n}\right) \in M,\left\langle p_{i}, p_{i}\right\rangle_{i} \leqslant c_{i}, i=1, \ldots, n, q_{j} \in \partial M_{j},\left\langle\nu_{q_{j}}, p_{j}\right\rangle_{j}>0\right\} .
\end{aligned}
$$

Let us denote by $V_{j}^{--}, j=1, \ldots, n$ the following set

$$
\begin{aligned}
V_{j}^{--}=\left\{0 \leqslant t \leqslant T,\left(q_{1}, p_{1}, \ldots, q_{n}, p_{n}\right) \in T M^{+}:\right. & \\
& \left.\left(q_{1}, \ldots, q_{n}\right) \in M,\left\langle p_{i}, p_{i}\right\rangle_{i} \leqslant c_{i}, i=1, \ldots, n,\left\langle\nu_{q_{j}}, p_{j}\right\rangle_{j} \geqslant 0, q_{j} \in \partial M_{j}\right\} .
\end{aligned}
$$

Since we assume (6), then $W^{--}$is compact:

$$
W^{--}=V_{1}^{--} \cup \ldots \cup V_{n}^{--} .
$$

Therefore, $W^{-}$is also compact and $W$ is a simple periodic segment over $[0, T]$. Clearly, $V_{j}^{--}$is homotopic to $M_{1} \times \ldots \times \partial M_{j} \times \ldots \times M_{n}$. Moreover, $V_{j_{1}}^{--} \cap \ldots \cap V_{j_{m}}^{--}$is homotopic to $M_{1} \times \ldots \times \partial M_{j_{1}} \times \ldots \times \partial M_{j_{m}} \times \ldots \times M_{n}$. 


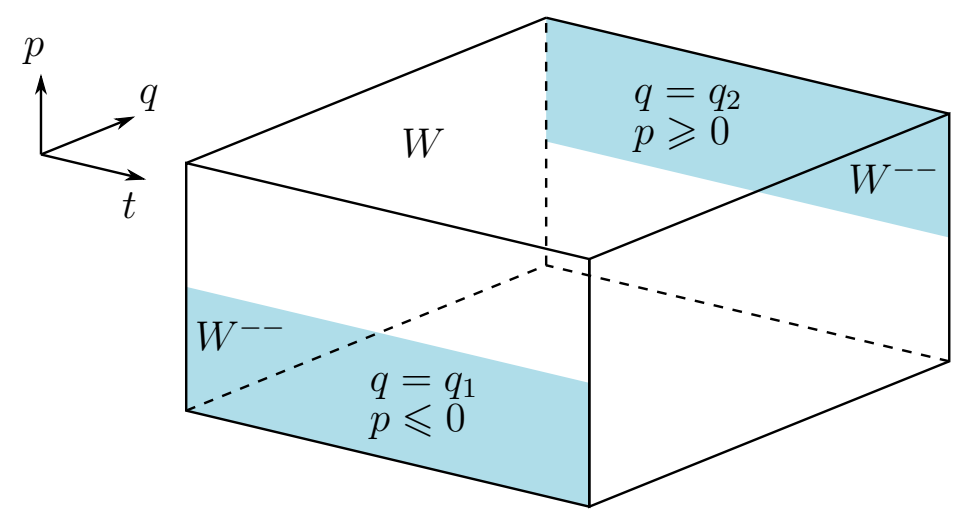

Figure 1: For a one-dimensional manifold parametrized by $q \in\left[q_{1}, q_{2}\right], W$ has the above form.

If $M_{i}$ has a nonempty boundary, then $\partial M_{i}$ consists either of a finite number of curves that are homeomorphic to circles $\left(\chi\left(\partial M_{i}\right)=0\right)$ or it is a two-pointed set $\left(\chi\left(\partial M_{i}\right)=2\right)$. Suppose, without loss of generality, that for some $k \chi\left(\partial M_{i}\right)=0$ iff $i>k$, i.e. there are $k \geqslant 0$ one-dimensional manifolds in $\left\{M_{i}\right\}$. From (8) we have

$$
\begin{aligned}
\chi\left(W^{--}\right) & =\sum_{1 \leqslant i \leqslant n} \chi\left(V_{i}^{--}\right)-\sum_{1 \leqslant i<j \leqslant n} \chi\left(V_{i}^{--} \cap V_{j}^{--}\right)+\ldots+(-1)^{n-1} \chi\left(V_{1}^{--} \cap \ldots \cap V_{n}^{--}\right) \\
& =\sum_{1 \leqslant i \leqslant k} \chi\left(V_{i}^{--}\right)-\sum_{1 \leqslant i<j \leqslant k} \chi\left(V_{i}^{--} \cap V_{j}^{--}\right)+\ldots+(-1)^{k-1} \chi\left(V_{1}^{--} \cap \ldots \cap V_{k}^{--}\right) \\
& =\chi\left(M_{1} \times \ldots \times M_{n}\right) \cdot\left(2 C_{k}^{1}-2^{2} C_{k}^{2}+\ldots+2^{k}(-1)^{k-1} C_{k}^{k}\right)=\chi(M) \cdot\left(1+(-1)^{k+1}\right) .
\end{aligned}
$$

Here we use that if $j_{1}<\ldots<j_{m} \leqslant k$, then

$$
\chi\left(M_{1} \times \ldots \times \partial M_{j_{1}} \times \ldots \times \partial M_{j_{m}} \times \ldots \times M_{n}\right)=2^{m} \chi\left(M_{1} \times \ldots \times M_{n}\right) .
$$

Finally, $\chi\left(W_{0}\right)=\chi(M)=\chi\left(M_{1}\right) \ldots \chi\left(M_{n}\right) \neq 0, \chi\left(W^{--}\right)=\chi\left(W_{0}^{-}\right)$. Therefore,

$$
\chi\left(W_{0}\right)-\chi\left(W_{0}^{-}\right) \neq 0
$$

and we can apply theorem 2.7 .

Let us now illustrate the theorem on examples.

Example 2.9. Consider a finite number of planar pendulums moving with viscous friction in a gravitational field (Fig. 2). Let $r_{i}$ be the radius-vector from a fixed origin to the massive point of the $i$-th pendulum. Suppose that the pivot points of the pendulums are moving along a horizontal line in accordance with a $T$-periodic law of motion $h: \mathbb{R} / T \mathbb{Z} \rightarrow \mathbb{R}$, which is the same for all pendulums. Let us also assume the following: for any two pendulums the distance between their pivot points is greater than the sum of their lengths, i.e. the pendulums are disjoint; for any two pendulums there is a repelling force $F_{i j}$ acting on the massive point of the $i$-th pendulum from the $j$-th pendulum $\left(F_{i j}\right.$ is parallel to $\left.r_{i}-r_{j}\right)$. 


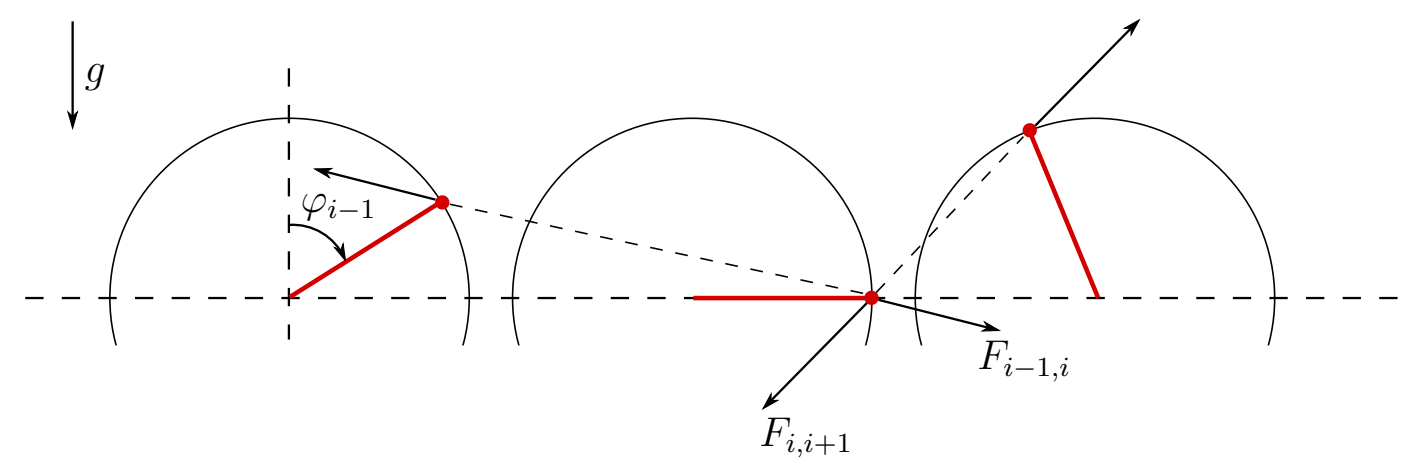

Figure 2: When the $i$-th pendulum is horizontal, the repelling forces acting on it are directed downward.

The equations of motion can be presented in the following form:

$$
\begin{aligned}
\dot{\varphi}_{i} & =p_{i}, \\
\dot{p}_{i} & =-\frac{\ddot{h}}{l_{i}} \cos \varphi_{i}+\frac{g}{l_{i}} \sin \varphi_{i}-\gamma_{i} p_{i}+\frac{1}{m_{i} l_{i}} \sum_{j \neq i}\left(F_{i j}, e_{\varphi_{i}}\right) .
\end{aligned}
$$

Here $m_{i}$ is the mass of the $i$-th massive point; $l_{i}$ is the length of the $i$-th pendulum; $\gamma_{i}>0$ is the viscous friction coefficient for the $i$-th pendulum (divided by the mass $m_{i}$ ); $e_{\varphi_{i}}$ is the following unit vector $e_{\varphi_{i}}=\left(\cos \varphi_{i},-\sin \varphi_{i}\right)$; for given $\varphi_{i}, \varphi_{j}, p_{i}, p_{j}$, we assume that

$$
F_{i j}\left(\varphi_{i}, p_{i}, \varphi_{j}, p_{j}\right)=\frac{r_{i}-r_{j}}{\left|r_{i}-r_{j}\right|} \cdot f_{i j}\left(\varphi_{i}, \varphi_{j}\right)
$$

where $f_{i j} \geqslant 0$ is a smooth real-valued function. Then there exists a $T$-periodic solution of (9).

Indeed, let $M_{i}$ be a compact manifold defined by the inequalities $-\pi / 2 \leqslant \varphi_{i} \leqslant \pi / 2$, $\chi\left(M_{i}\right)=1$. From $(9)$ it follows that

$$
\begin{aligned}
& \left.\dot{p}_{i}\right|_{\varphi_{i}=\pi / 2, p_{i}=0}=\frac{g}{l_{i}}+\left.\frac{1}{m_{i} l_{i}} \sum_{j \neq i}\left(F_{i j}, e_{\varphi_{i}}\right)\right|_{\varphi_{i}=\pi / 2}>0, \\
& \left.\dot{p}_{i}\right|_{\varphi_{i}=-\pi / 2, p_{i}=0}=-\frac{g}{l_{i}}+\left.\frac{1}{m_{i} l_{i}} \sum_{j \neq i}\left(F_{i j}, e_{\varphi_{i}}\right)\right|_{\varphi_{i}=-\pi / 2}<0 .
\end{aligned}
$$

Therefore, theorem 2.8 can be applied. Let us also note that along the obtained periodic solution we always have $\varphi_{i} \in(-\pi / 2, \pi / 2)$, i.e. the pendulums always remain above the horizontal line.

Remark 2.10. Note that if in the previous example we replace the half-circles by any compact manifolds with boundaries such that their Euler-Poincaré characteristics are non-zero, the boundaries are in a horizontal plane, the rest of the manifolds are above the horizontal plane and they are vertical at the boundaries, then, for such a system, there also exists a periodic solution. Moreover, we can consider arbitrary external periodic fields acting on the massive points instead of the periodic motion of the surfaces. If the external fields are directed downward at the horizontal plane then there exists a periodic solution (Fig. 3). 


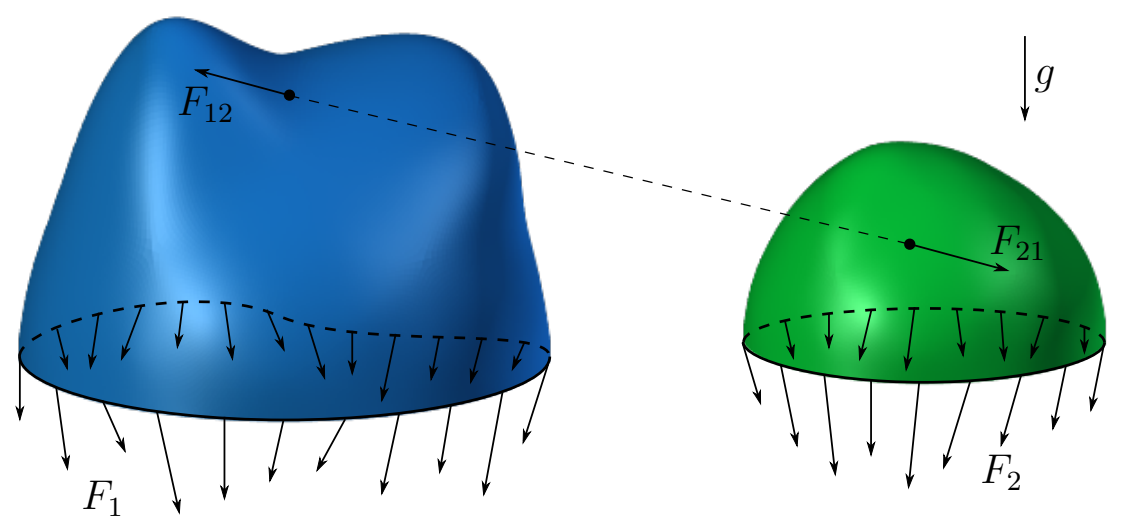

Figure 3: The points are repelling; the external fields $F_{1}$ and $F_{2}$ are periodic and directed downward at the boundaries.

Remark 2.11. One can also consider systems of coupled pendulums in which the pendulums attract each other. An example of such a system is presented in the figure below (Fig. 4). Here we assume that the massive points of the pendulums are charged, they interact with the Coulomb interaction and the pendulums are located in a parallel electric field $E$. This system is similar to example 2.9 and also has a periodic solution.

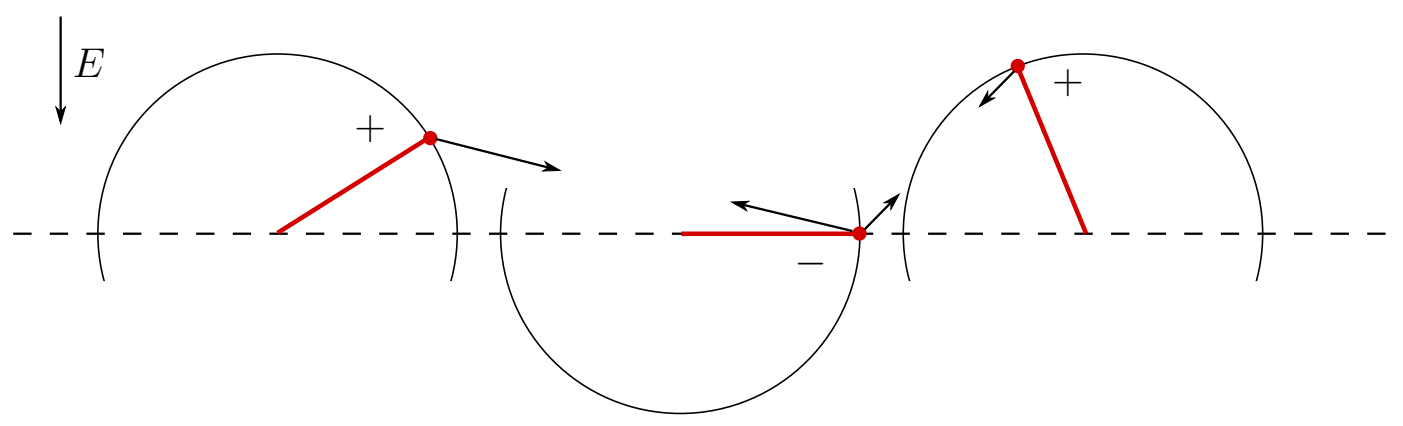

Figure 4: An example of the system with a periodic solution. The pendulums attracts each other and their pivot points are moving periodically along the horizontal line.

Example 2.12. Consider a chain of coupled oscillators in an external periodic field. We suppose that the oscillators are located along a straight line, i.e. every oscillator has at most two neighbours. For the boundary oscillators, which have only one neighbour, we assume that the corresponding massive points are fixed. We also assume that the oscillators are moving with viscous friction and the interaction between them is described by a Morse potential (Fig. 5). For simplicity, let $m_{i}=1$ and $\gamma>0$ is the same viscous friction coefficient for all oscillators. The equations of motion can be written as follows:

$$
\ddot{x}_{i}=-\gamma \dot{x}_{i}-\frac{\partial V\left(x_{i}-x_{i-1}\right)}{\partial x_{i}}-\frac{\partial V\left(x_{i+1}-x_{i}\right)}{\partial x_{i}}+F\left(t, x_{i}\right), \quad i=1, \ldots, n,
$$

where

$$
V: \mathbb{R} \rightarrow \mathbb{R}, \quad V(x)=\frac{1}{2}\left(1-e^{-(x-\delta)}\right)^{2},
$$


$F: \mathbb{R} / T \mathbb{Z} \times \mathbb{R} \rightarrow \mathbb{R}$ and $\gamma>0, \delta>0, x_{0}=0$ and $x_{n+1}=(2 n+1)(\delta+a), a \geqslant \ln 2$ is a parameter.

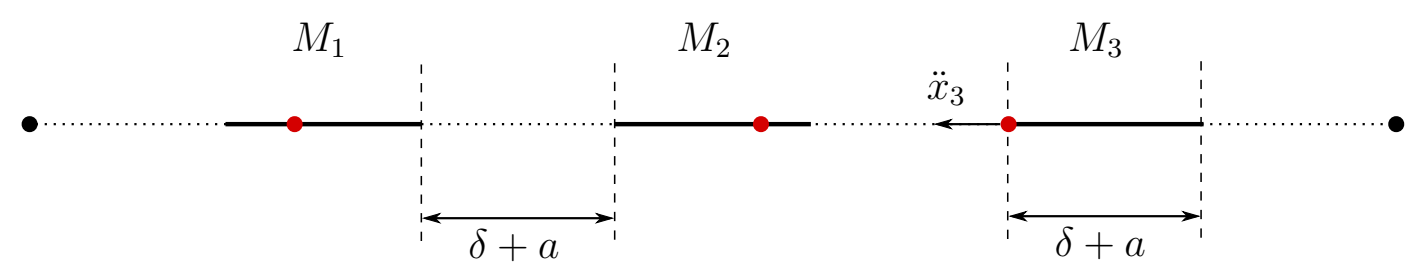

Figure 5: For $n=3$ we have three compact manifolds with boundaries. The manifolds $M_{i}$ are distanced from each other so the oscillators attract one another; $\ddot{x}_{i}$ is directed outward at the boundary $\partial M_{i}$.

Let us now show that if the function $F$ satisfies the following condition

$$
(-1)^{k+1} \cdot F(t, k(\delta+a))<0, \text { for all } t \in \mathbb{R} / T \mathbb{Z}, k=1, \ldots, 2 n,
$$

then the system (11) has a periodic solution. Indeed, let $M_{i}$ be the following compact manifold with a boundary

$$
M_{i}=\left\{x_{i} \in \mathbb{R}:(2 i-1)(\delta+a) \leqslant x_{i} \leqslant 2 i(\delta+a)\right\} .
$$

Since $\left.\frac{\partial^{2} V}{\partial x^{2}}\right|_{x=\delta+\ln 2}=0$ and $\frac{\partial^{2} V}{\partial x^{2}}<0$ for $x>\delta+\ln 2$, then

$$
\left.\frac{\partial V}{\partial x}\right|_{x=x_{2}} \leqslant\left.\frac{\partial V}{\partial x}\right|_{x=x_{1}} \quad \text { if } \quad \delta+\ln 2 \leqslant x_{1} \leqslant x_{2} .
$$

Finally, taking into account (12), we obtain that $\ddot{x}_{i}$ is directed outward to $M_{i}$ at the boundary $\partial M_{i}$ provided $\dot{x}_{i}=0$ and 2.8 can be applied.

\section{Conclusion}

Taking into account that the presented approach for studying forced oscillations is relatively simple, and, at the same time, it allows one to use a wide range of 'building blocks' and types of forces to construct a physical system that has a periodic solution, we hope that our result will be useful in applications.

\section{References}

[1] G. Hamel, Über erzwungene Schwingungen bei endlichen Amplituden, in: Festschrift David Hilbert zu Seinem Sechzigsten Geburtstag am 23. Januar 1922, Springer, 1922, pp. 326-338.

[2] V. Benci, M. Degiovanni, Periodic solutions of dissipative dynamical systems, in: Variational Methods, Springer, 1990, pp. 395-411.

[3] M. Furi, Second order differential equations on manifolds and forced oscillations, in: Topological Methods in Differential Equations and Inclusions, Springer, 1995, pp. 89-127.

[4] M. Furi, M. P. Pera, The forced spherical pendulum does have forced oscillations, in: Delay differential equations and dynamical systems, Springer, 1991, pp. 176-182. 
[5] M. Furi, M. Pera, On the existence of forced oscillations for the spherical pendulum, Boll. Un. Mat. Ital. 4 (2) (1990) 381-390.

[6] J. Mawhin, Global results for the forced pendulum equation, Handbook of Differential Equations: Ordinary Differential Equations 1 (2000) 533-589.

[7] J. Marlin, Periodic motions of coupled simple pendulums with periodic disturbances, International Journal of Non-Linear Mechanics 3 (4) (1968) 439-447.

[8] J. Mawhin, Une généralisation de théorèmes de J.A. Marlin, International Journal of Non-Linear Mechanics 5 (2) (1970) 335-339.

[9] P. Drábek, S. Invernizzi, Periodic solutions for systems of forced coupled pendulum-like equations, Journal of differential equations 70 (3) (1987) 390-402.

[10] Y. V. Kartashov, B. A. Malomed, L. Torner, Solitons in nonlinear lattices, Reviews of Modern Physics 83 (1) (2011) 247.

[11] M. Toda, Theory of nonlinear lattices, Vol. 20, Springer Science \& Business Media, 2012.

[12] S. Flach, C. R. Willis, Discrete breathers, Physics reports 295 (5) (1998) 181-264.

[13] J. Marin, S. Aubry, Breathers in nonlinear lattices: numerical calculation from the anticontinuous limit, Nonlinearity 9 (6) (1996) 1501.

[14] I. Polekhin, Forced oscillations of a massive point on a compact surface with a boundary, Nonlinear Analysis: Theory, Methods \& Applications Volume 128 (2015) 100-105.

[15] R. Srzednicki, K. Wójcik, P. Zgliczyński, Fixed point results based on the Ważewski method, in: Handbook of topological fixed point theory, Springer, 2005, pp. 905-943.

[16] T. Wazewski, Sur un principe topologique de l'examen de l'allure asymptotique des intégrales des équations différentielles ordinaires, Ann. Soc. Polon. Math 20 (1947) 279-313. 